\title{
Performance of Recent Optimization Algorithms and Its Comparison to State-of-the-Art Differential Evolution and Its Variants for the Economic Optimization of Cooling Tower
}

\author{
Patel, Vivek K.; Raja, Bansi D.; Savsani, Vimal J.; Desai, Nishith B.
}

Published in:

Archives of Computational Methods in Engineering

Link to article, DOI:

10.1007/s11831-021-09529-2

Publication date:

2021

Document Version

Peer reviewed version

Link back to DTU Orbit

Citation (APA):

Patel, V. K., Raja, B. D., Savsani, V. J., \& Desai, N. B. (2021). Performance of Recent Optimization Algorithms and Its Comparison to State-of-the-Art Differential Evolution and Its Variants for the Economic Optimization of Cooling Tower. Archives of Computational Methods in Engineering, 28, 4523-4535. https://doi.org/10.1007/s11831-021-09529-2

\section{General rights}

Copyright and moral rights for the publications made accessible in the public portal are retained by the authors and/or other copyright owners and it is a condition of accessing publications that users recognise and abide by the legal requirements associated with these rights.

- Users may download and print one copy of any publication from the public portal for the purpose of private study or research.

- You may not further distribute the material or use it for any profit-making activity or commercial gain

- You may freely distribute the URL identifying the publication in the public portal 


\title{
Performance of recent optimization algorithms and its comparison to state- of-the-art Differential evolution and its variants for the economic optimization of cooling tower
}

\author{
Vivek K. Patel* \\ Department of Mechanical Engineering \\ School of Technology, Pandit Deendayal Petroleum University, Gandhinagar, Gujarat, India \\ E-mail: vivekp@,sot.pdpu.ac.in
}

\section{Bansi D. Raja}

Department of Mechanical Engineering,

Indus University, Ahmedabad, Gujarat, India

E-mail: bansi14.raja@gmail.com

\author{
Vimal J. Savsani \\ Department of Engineering, \\ Canadore College, North Bay, ON, Canada \\ E-mail: vimal.savsani@canadorecollege.ca
}

\section{Nishith B. Desai}

Department of Mechanical Engineering,

Technical University of Denmark, 2800 Kongens Lyngby, Denmark

E-mail : nbdes@mek.dtu.dk

* Corresponding author 


\begin{abstract}
Present work explores the quantitative and qualitative performance investigation of nine recently developed optimization algorithm along with state-of-the-art differential evolution and its variants for the economic optimization of cooling tower. Quantitative performance of the competitive algorithms evaluated based on the best solution, mean solution, success rate, and standard deviation results. Qualitative performance comparison of competitive algorithms obtained through statistical analysis of the obtained results. Statistical analysis is carried out with two different scenarios to identify the qualitative performance of recently developed algorithms alone and in the presence of stat-of-art differential evolution and its variants. The effect of different constraint handling methods on the performance of the competitive algorithms is identify and presented. The behaviour of all competitive algorithms is verified for sensitiveness of constraints. Finally, the convergence behaviour of the competitive algorithm in obtaining the minimum total cost solutions is also obtainable and discussed.
\end{abstract}

\title{
Key words
}

Optimization algorithms; Comparative performance; Statistical analysis; Convergence comparison; Constraint handling methods

\section{Introduction}

In the last demi-decade, researchers proposed variety of evolutionary optimization algorithms for addressing the optimization problems associated with different field of science and engineering. These algorithms are inspired from the various natural phenomenon such as social behaviour of swarms, biology evolution, laws of natural, laws of chemical or physics etc. These algorithms was experimented on the various constraint and unconstraint 
benchmark functions by the developers to prove the effectiveness of their algorithms. At later stage, these algorithms also applied for the optimization of different engineering and science application by the researchers across glob. In addition to that modified and hybrid versions of these algorithms are also proposed by the researchers and experiment on the various real life optimization problem. Performance of the modified and hybrid version of these algorithms seems to be improved as compared to the basic version.

Few such evolutionary algorithms developed in the recent past are Squirrel search algorithm (SSA) [1], Harris hawks optimization algorithm (HHO) [2], Marine Predators Algorithm (MPA) [3], Grey wolf optimizer (GWO) [4], Equilibrium optimizer (EO) [5], Sailfish Optimizer (SFO) [6], Spotted hyena optimizer (SHO) [7], Henry gas solubility optimization (HGSO) [8], Pathfinder algorithm (PFA) [9], Salp swarm (SS) algorithm [10], Cuckoo search algorithm (CSA) [11], Heat transfer search (HTS) [12], Water wave optimization (WWO) [13], Yin-Yang-pair Optimization [14], Ant lion optimizer (ALO) [15], Artificial flora (AF) algorithm [16], Sine-cosine algorithm (SCA) [17], Dragonfly algorithm (DA) [18], Electro-search (ES) algorithm [19], Mouth brooding fish (MBF) [20] etc. The researcher proposed these algorithms with the aim of enhancing the performance of evolutionary computation. The literature survey revel that these algorithms applied for the optimization of various engineering application belonging to mechanical, civil, electrical, chemical, computer and other allied branches.

For the assessment of the evolutionary optimization algorithm, a competition known as "Congress on Evolutionary Computation" (CEC) is organised almost every year [21]. A common platform provided in CEC to experiment and compares the results of various evolutionary algorithms to identify the winner algorithm. However, it can be observed from the literature survey that majority of the evolutionary algorithms developed in the last demidecade were not a winner algorithm of the CEC competition. In fact, majority of these 
algorithms even not participated in this competition. On the other hand, these algorithms applied successfully on various real life optimization problems. The state-of-the-art evolutionary optimization algorithms such as Differential evolution (DE) [22] and its variants like Self adaptive DE (SaDE) [23], linear population size reduction DE (L-SHADE) [24] etc. quite often win the CEC competition. However, the literature revel the quite few study involving the comparative performance of these state-of-the-art optimization algorithms and various recently developed algorithms for the optimization of engineering applications.

Cooling towers are one of the important energy conservation equipment used in power plant. Cooling tower is used to cool the condenser water of the power plant. In general, the cost effective design of cooling tower can be achieved by optimizing its operating variables [25]. These variables includes continuous, discrete, and integer characteristic. Additionally, the optimized geometry should satisfy various constraints having linear and nonlinear characteristic. The optimization problem of cooling tower is multi-model in nature. Further, the numbers as well as types of variables and constraints are also change with the type of cooling tower. Application of few established algorithms like GA, DE, PSO, and $\mathrm{ABC}$ for the economic optimization of cooling tower is available in literature. For instant, Singh and Das $[26,27]$ adapted genetic algorithm (GA) for the parameter optimization of the cooling tower. Rao and Patel [28] implemented artificial bee colony (ABC) algorithm for the optimization of mechanical draft cooling tower. Ozcan et al. [29] put an effort to optimize the cost of air cooling system of a cooling tower through Particle swarm optimization (PSO) and DE algorithms. On the other side, majority of evolutionary algorithms developed in the last demi decades yet not explore for the cooling tower optimization. Hence, the comparative performance of such recently developed algorithms with state-to-art algorithm yet not observed in the literature. 
In the proposed work, nine recently developed optimization algorithms are investigated and their performances are measured with respect to state-of-the art $\mathrm{DE}, \mathrm{SaDE}$,

and L-SHADE. The main purpose of the present work are (i) to evaluate effectiveness of recently developed algorithms such as EO, SFO, SSA, SHO, HHO, MPA, PFA, MBF, and HGSO along with stat-of-art DE, SaDE, and L-SHADE for the economic optimization of cooling tower (ii) performance comparison of the considered recently developed algorithm with stat-of-art DE and its variant (iii) evaluate the effect of different constraint handling methods on the performance of comparative algorithms (iv) identify the behaviour of all competitive algorithms for sensitiveness of constraints. (v) assess the convergence performance of the competitive algorithms. Ability of each algorithm is demonstrated by attempting the case study of mechanical draft counter flow wet cooling tower. The organisation of the remaining paper includes brief description of recently developed and state-of-the-art metaheuristic (Section 2); objection function formulation and constraints description (Section 3); attempt case study and result-discussion (Section 4); and finally the conclusion of the present investigation describe in section 5 .

\section{Recently developed and state-of-the-art optimization algorithms}

This section presents the brief description of the recently developed metaheuristic along with state-of-the-art DE, SaDE, and L-SHADE experimented in this work. The principal aspect of each algorithm is present in this section. The readers can get the detail description and working of the particular algorithms from the mention references.

\subsection{Spotted hyena optimizer}

Dhiman and Kumar [7] proposed the spotted hyena optimizer (SHO) algorithm. The SHO inspired from the intelligent behaviour of spotted hyena and mimic the collaborative hunting behaviour of spotted hyenas. The hunting behaviour of the spotted hyena consists of 
three basic steps and so as the SHO algorithm. These three steps of the SHO algorithm mimic the searching, encircling, and attacking prey behaviour of the spotted hyena.

\subsection{Mouth brooding fish algorithm}

Jahani and Chizari [20] proposed the mouth brooding fish (MBF) algorithm. The MBF is swarm intelligence based metaheuristic. The MBF algorithm inspire from the movement of one of the underwater creature 'mouth brooding fish'. The MBF algorithm simulates the intelligent behaviour of the mouth brooding fish to protect their offspring. This intelligent behaviour includes movement, dispersion and protection of mouth brooding Fish.

\subsection{Squirrel search algorithm}

Jain et al. [1] proposed the squirrel search algorithm (SSA). The SSA inspired from the intelligent foraging behaviour of southern flying squirrels. The SSA mimics the food searching, storage and utilization ability of the flying squirrel exhibit in the different climate throughout their lifecycle. The SSA mathematically modelled the dynamic foraging strategy and gliding mechanism of flying squirrels.

\subsection{Sailfish optimizer}

Sailfish Optimizer (SFO) is a population oriented metaheuristic algorithm proposed by Shadravan et al. [6]. The SFO algorithm inspired from the group hunting behaviour of sailfish. The SFO algorithm simulated the search, attack-alternation, hunting and catching prey behaviour of sailfish. The SFO consists of two tips of populations, sailfish population and sardines population.

\subsection{Pathfinder algorithm}

Yapici and Cetinkaya [9] proposed the pathfinder algorithm (PFA). The PFA imitate cooperative movement of animal group. The algorithm simulates the leadership behaviour and social interaction of the animal group in search of food area or prey. Every individual in 
the animal group changes their position and follow the pathfinder during the process of food search.

\subsection{Harris hawks optimization}

Heidari et al. [2] presented harris hawks optimization (HHO) algorithm. The HHO algorithm inspired from the supportive behaviour and chasing style of Harris' hawks bird of prey. In nature, several harris hawks pounce and chase prey from different direction considering the escaping pattern of prey. The HHO algorithm simulates the seven skill strategy of harris hawks to hunt and capture a prey.

\subsection{Henry gas solubility optimization}

Hashim et al. [8] proposed henry gas solubility optimization (HGSO) algorithm. The HGSO is a physics law based algorithm that imitates Henry's law of gas solubility. The Henry's law correlate amount of gas dissolved in a given volume of liquid at constant temperature. The HGSO algorithm simulates the same huddling behaviour of gas mathematically to form optimization algorithm.

\subsection{Marine predator algorithm}

Faramarzi et al. [3] proposed marine predator algorithm (MPA). The MPA algorithm inspired from the foraging strategy of marine predators. The marine predator exhibit two policies namely Lévy and Brownian motion for foraging. The foraging process of the marine predators is based on the three conditions depending on the velocity ratio of the predator and prey. Likewise, the MPA algorithm is also working in three phases.

\subsection{Equilibrium optimizer}

The Equilibrium optimizer (EO) algorithm, proposed by Faramarzi et al. [5], is a metaheuristic inspired by the conservation of mass theory. The EO algorithm simulates the mass balance principles applied to any control volume in order to evaluate the unknown concentration of a constituent. The search mechanism of the EO algorithm depends on the 
three important term know as equilibrium concentration (belonging to equilibrium pool), concentration difference, and generation rate.

\subsection{Differential evolution algorithm}

Storn and Price [22] proposed differential evolution (DE) algorithm. The algorithm works with control parameters namely crossover, mutation, and selection operator. The algorithm performs the exploration of the search space through mutation operation and exploitation through selection operation. Further, the DE algorithm employed greedy selection operator for the fast convergence rate.

\subsection{Self-adaptive differential evolution}

Qin and Suganthan [23] incorporate the self-adaptation strategy in the DE algorithm and proposed self-adaptive differential evolution (SaDE) algorithm. The learning strategy and control parameter of DE algorithm are self-adapted in the SaDE variant based on the previous learning experience. This behaviour of SaDE algorithm makes it highly consistent and produces promising results on variety of problems having different characteristics.

\subsection{Linear population size reduction differential evolution}

Linear population size reduction differential evolution (L-SHADE) is an improved version of success-history based adaptive differential evolution (SHADE) algorithm proposed by Tanabe and Fukunaga [24]. L-SHADE used linear population size reduction to reduce the population linearly with function evaluation. The population size reduction strategy enhances the performance of L-SHADE very effectively. The L-SHADE had been successfully applied to various CEC benchmark problems.

\section{Objective function formulation and constraints description}

Typically the cooling tower is used to cool the condensing water in the power plant. Majority, mechanical draft cooling towers are used for such purpose. Present work focused 
on the economic optimization of cooling tower. Minimization of total annual cost $\left(C_{t o t}\right)$ is treated as objective function for the economic optimization. Total annual cost of the cooling tower composed of two components namely annualized capital cost $\left(C_{c a p}\right)$ and operating cost $\left(C_{o p}\right)$. The annualized capital cost of the cooling tower composed of fixed cost $\left(C_{f}\right)$ and incremental cost. The incremental cost of the cooling tower depends on the fill volume and air mass flow rate. Considering the above mention parameters, annualized capital cost of the cooling tower is obtained through below equation $[25,30]$,

$C_{\text {cap }}=C_{f}+\left[\left(C_{f v} * A_{f i} * L_{f i}\right)+\left(C_{m a} * m_{a}\right)\right]$

Where, $C_{f}$ is the fixed cost, $C_{f v}$ is the incremental cost of cooling tower per unit volume, and $C_{m a}$ is the incremental cost per unit mass flow rate of air. $A_{f i}$ the tower fill area, $L_{f i}$ is the fill height of the cooling tower and $m_{a}$ is the mass flow rate of the air.

Likewise, the operating cost of the cooling tower depends on the pumping power and makeup water cost and obtained through below equation $[25,30]$,

$$
C_{o p}=\left(P * C_{e}+m_{m w} * C_{w}\right) * Y_{h r}
$$

Where, $C_{e}$ and $C_{w}$ is the cost of the electricity and makeup water cost. Also, $P$ is the pumping power, $m_{m w}$ is the makeup water requirement, and $Y_{h r}$ is the yearly operating hours.

Based on the capital cost and operating cost, the total annual cost is obtained by $[30,12]$

$$
C_{t o t}=a_{c c} * C_{c a p}+C_{o c}
$$

Where, $a_{c c}$ is the annualised factor. The details mathematical formulation of each of the above terms can be found in Appendix.

Further, the economical optimized geometry of cooling tower should satisfy all the associated design constraints. To ensure that constraints are satisfied during the optimization, adaptive penalty method is used in the present work. In fact, various constraints handling 
method experimented in the proposed work to identify its effect on the optimization algorithms. So, the objective function for the cooling tower optimization is presented as,

$$
\text { Minimize } f(x)=C_{\text {tot }}+\sum_{j=1}^{m} z_{j} \bar{g}_{j}(x)
$$

Where, the second term specifies the adaptive penalty function and defined as,

$z_{j}=\left|\overline{C_{t o t}}\right|+\frac{\bar{g}_{j}(x)}{\sum_{j=1}^{m}\left[\bar{g}_{j}(x)\right]^{2}}$

Where, $\bar{g}_{j}(x)$ are the associate constraints of the cooling tower.

The above mention objective function for cooling tower subjected to following constraints.

\subsection{Temperature constraints}

Ideally the minimum outlet water temperature from the cooling tower is the atmospheric air wet bulb temperature $\left(W B T_{\text {air,in }}\right)$. However, the cooling water outlet temperature $\left(T_{\text {water,out }}\right)$ is always more than the wet bulb temperature of atmospheric air. In the present work, this temperature difference is considered as $2.8^{\circ} \mathrm{C}$ or more than it.

$T_{\text {water,out }}-W B T_{\text {air }, \text { in }} \geq 2.8$

Likewise, the cooling water outlet temperature should be less than the hot process fluid temperature $\left(T_{p f}\right)$ of the condenser where the cooling water used. In the present work, this temperature difference should be more than $5^{\circ} \mathrm{C}$.

$T_{p f}-T_{\text {water,out }} \geq 5$

Also, to ensure proper heat transfer, the water stream should be cooled and temperature of air stream should be increases in the cooling tower.

$$
\begin{aligned}
& T_{\text {water,in }}-T_{\text {water,out }} \geq 0 \\
& T_{\text {air,out }}-T_{\text {air,in }} \geq 0
\end{aligned}
$$


Where, $T_{\text {water }}$ and $T_{\text {air }}$ is the water and air temperature while subscript in and out stand for inlet and outlet condition respectively.

\subsection{Merkel number constraint}

Merkel number $(\mathrm{Me})$ represents the driving force required for proper heat transfer between air and water at any section of cooling tower. The Merkel number depends on the water temperature and air enthalpy at any given section. In the proposed work, to ensure the proper driving force for the heat transfer, the Merkel number should be 5 or more than it.

$M e=\int_{\mathrm{T}_{\text {water,out }}}^{\mathrm{T}_{\text {water,in }}} \frac{c_{p w} d T_{\text {water }}}{h_{s a}-h_{a}} \geq 5$

Where, $h_{a}$ is the enthalpy of air and $h_{s a}$ is the enthalpy of saturated air. $c_{p w}$ denotes the specific heat of water.

\subsection{Tower fill area constraint}

Tower fill area $\left(A_{f i}\right)$ represent the area packed by the fill material to enhance the heat transfer between air and water. However, the pressure drop of air and water increases with the fill area which further increases the operating cost. In the present work, the tower fill area should be $10 \mathrm{~m}^{2}$ or less than it.

$A_{f i} \leq 10$

\subsection{Pressure drop constraint}

The major pressure drop in the cooling tower is due to fill material $\left(\Delta P_{f i}\right)$. The other miscellaneous pressure drop $\left(\Delta P_{\text {misc }}\right)$ includes loss due to air entry and exit, drift eliminators, water distributing arrangement etc. In the proposed work, the total pressure drop $\left(\Delta P_{t}\right)$ of the cooling tower should not more than $230 \mathrm{~Pa}$.

$\Delta P_{t}=\left(\Delta P_{f i}+\Delta P_{m i s c}\right) \leq 230$ 


\section{Case study and results-discussion}

A case study of mechanical draft counter flow cooling tower is attempted in the proposed work to assess the performance comparison of recently developed metaheuristic such as EO, SFO, SSA, SHO, HHO, MPA, PFA, MBF, HGSO with state-of-the-art differential evolution and its variants. All the algorithms executed with common platform on the considered case study. Further, to get a more insight on performance comparison, the recently developed algorithm assess with and without presence of stat-of-art differential evolution and its variants.

\subsection{Case study}

A mechanical draft cooling tower need to design based on economic optimization. Fig. 1 represents the schematic of one such cooling tower [25]. The proposed cooling tower should fulfil the heat duty of 3.4 MW. The hot cooling water from the condenser enters into the cooling tower at $50^{\circ} \mathrm{C}$. The desired outlet temperature of the water from cooling tower is $25^{\circ} \mathrm{C}$. The atmospheric air enters into cooling tower at $22^{\circ} \mathrm{C}$. The wet bulb temperature of the air is $12^{0} \mathrm{C}$. Inlet temperature of the hot process fluid in condenser is $65^{\circ} \mathrm{C}$ which should be cooled to $30^{\circ} \mathrm{C}$ by using cooling water from the cooling tower.

The economic optimization of cooling tower depends on the different geometric and operating parameters. Three such variables which combine the effect of geometric and operating parameters such as tower fill area, mass of water, and mass of air are considered in the present work. The lower and upper bound of these variables along with the other economic parameters required to evaluate the total cost of the cooling tower are shown in Table 1. Further, the optimized geometry of cooling tower should satisfy the temperature, pressure drop, fill area, and Merkel number constrains mentioned previously. 


\subsection{Results and discussion}

All the algorithms considered in this work are executed with population size of 50 and 100000 maximum function evaluation. The control parameters of each algorithm are mention in Table 2. Further, each algorithm executed 100 times on the consider case study of cooling tower to analyse its comparative performance. Moreover, $0.01 \%$ variation from the minimum cost value is considered for obtaining the success rate of each competitive algorithm.

The quantitative performance comparison of each considered algorithm for cooling tower optimization is shown in Table 3. The results are displayed in the form of best value, worst value, and mean value along with the success rate, and standard deviation obtained from the 100 run of each competitive algorithm. In this comparative result, best value is the minimum value obtained by the particular algorithm. Mean value is the average value of objective function over 100 independent run of the particular algorithm and used to judge its average performance.

The comparative result indicates that SHO, SSA, HHO, EO, DE and both its variants (i.e. SaDE, L-SHADE) produces the identical solution in terms of minimum cost and leads towards the economically optimized design of cooling tower. However, the average performance of DE, SaDE, and L-SHADE are better than other comparative algorithm. The SSA and EO algorithm follows the DE and its variants in terms of average performance. Likewise, with 97 and 98\% success rate, the DE and its variants perform superior than other approaches in terms of success rate comparison. The SSA, EO, HHO, and SHO algorithm also produces success rate more than $90 \%$. In terms of standard deviation, again DE and its variants performed better with less spread out of the solutions followed by the EO and SSA. Thus, the quantitative performance of DE and its variants dominant the other recently developed algorithms in almost all the comparison criteria. 
The qualitative performance of the considered algorithms is evaluated by implementing the statistical analysis of the comparative results. The statistical analysis is performed with two different scenarios. In first scenario, the results of recently developed algorithms are considered only. In second scenario the results of DE, SaDE, and L-SHADE are considered along with the recently developed algorithms. Further, the ranking of the competitive algorithms are also given based on its statistical performance. The statistical performance of the competitive algorithms is evaluated by executing the Friedman test [31] on the obtained results.

Table 4 presents the result of Friedman rank test for first scenario. For better insight and interpretation, the normalized value of Friedman test is also presented in Table 4. The normalized value of Friedman test is used to obtain the rank of the algorithms. The normalized value ' 1 ' shows the best performing algorithm. The algorithms having normalized value away from ' 1 ' indicates that its performance is degraded as compared to best algorithm. Further, the graphical presentation of Friedman rank test is also shown in Fig. 2. One can observe from the results that SSA obtained $1^{\text {st }}$ rank followed by the EO and HHO algorithm. Likewise, Table 5 presents the result of Friedman rank test for second scenario while its graphical presentation is shown in Fig. 3. It can observe from the results that L-SHADE obtained $1^{\text {st }}$ rank followed by the SaDE and DE algorithm. Further, there were significant difference in normalized value of considered recently developed algorithm and DE \& its variants. Thus, DE, SaDE, and L-SHADE outperform the considered recently developed algorithms for cooling tower optimization problem.

The present work of cooling tower optimization deals with various operating and geometric constraints. These constraints are taken care by constraint handling method. So, different constraints handling methods are experimented to identify its effect on the performance of the each competitive algorithm. Five different constraint handling methods 
[32] namely static penalty, adaptive penalty, $\varepsilon$-constraint, stochastic ranking, and superiority of feasible solutions method are experimented with the each of the competitive algorithm. Here also, the stopping criteria of the algorithm is set as 100000 function evaluation and 100 runs of all algorithm executed with each constrained handling method. The results obtained by each algorithm with different constraint handling methods are presented in Table 6 . The results are presented in terms of best and mean value along with the standard deviation obtained in 100 runs. The result shows that DE, SaDE, and L-SHADE perform better than the other algorithms with each of the constraint handling methods considered in this work. Further, the performance of adaptive penalty, stochastic ranking, and $\varepsilon$-Constraint method is almost similar with all the competitive algorithms for the cooling tower optimization.

The optimized design variables of cooling tower along with the total cost and the constraints value obtained using the L-SHADE is presented in Table 7. Results show that all the constraints are satisfied in the optimized design. Further, Fig. 4 shows the convergence performance of each competitive algorithm in obtaining minimum total cost design. The detail convergence behaviour of each competitive algorithm at the interval of specific number of function evaluations are shown in Fig, 5. It can be observed from Fig. 4 and 5 that the DE, SaDE, and L_SHADE converge within about 10000-15000 function evaluation. Other competitive algorithms converge within about 25000-50000 maximum function evaluations to their respective objective function value. Thus, convergence performance of the DE and its variants is better than rest of the approaches.

Finally, the behaviour of all competitive algorithms is verified for sensitiveness of constraints. Tower fill area and Merkel number constraints are considered for this investigation as both constraints are sensitive for economic cooling tower design. Five different value of tower fill area $\left(9.6 \mathrm{~m}^{2}, 9.8 \mathrm{~m}^{2}, 10 \mathrm{~m}^{2}, 10.2 \mathrm{~m}^{2}\right.$, and $\left.10.4 \mathrm{~m}^{2}\right)$ and Merkel number $(4.5,4.75,5,5.25$, and 5.5) are considered during the experimentation. The 
considered algorithms experimented 100 times for each constraint and average result obtained through the 100 independent runs presented to evaluate comparative performance. Table 8 and Table 9 show the performance of considered algorithms for various fill area and Merkel number constraints respectively. It can be observed from the results that $\mathrm{DE}, \mathrm{SaDE}$, and L-SHADE respond better than other competitive algorithms for constraints variation. Accordingly, the performance of DE, SaDE, and L-SHADE dominants the other approaches and produces the economically optimized cooling tower design in different scenario.

\section{Conclusion}

Solving applied problem of economic optimization is considered as a real test for the optimization algorithms. The present work demonstrates the performance comparison of nine recently developed algorithms such as EO, SFO, SSA, SHO, HHO, MPA, PFA, MBF, and HGSO with state-of-the-art DE, SaDE, and L-SHADE for the economic optimization of cooling tower. Quantitative results shows that DE, SaDE, and L-SHADE are better than recently developed algorithms considered in the present work followed by SSA and EO algorithms. The qualitative performance comparison of the obtained results also shows the dominance of DE and its variants. The L-SHADE achieves the $1^{\text {st }}$ rank followed by SaDE and DE. Further, the various constraint handling methods investigated with the considered algorithms and results shows that adaptive penalty, stochastic ranking and $\varepsilon$-constraint methods works equally well for the economic optimization. Convergence behaviour of DE, SaDE and L-SHADE algorithms are better than the other competitive algorithms in obtaining the optimum solution. Finally, DE and its variants respond better than other competitive algorithms for sensitiveness of constraints and dominants the other approaches of the present work. 


\section{References}

[1] M. Jain, V. Singh, A. Rani, A novel nature-inspired algorithm for optimization: Squirrel search algorithm, Swarm and evolutionary computation 44 (2019) 148-175.

[2] A.A. Heidari, S. Mirjalili, H. Faris, I. Aljarah, M. Mafarja, H. Chen, Harris hawks optimization: Algorithm and applications, Future Generation Computer Systems 97 (2019) 849-872.

[3] A. Faramarzi, M. Heidarinejad, S. Mirjalili, A.H. Gandomi, Marine Predators Algorithm: A Nature-inspired Metaheuristic, Expert Systems with Applications 152 (2020) 113377

[4] S. Mirjalili, S.M. Mirjalili, A. Lewis, Grey wolf optimizer. Advances in Engineering Software 69 (2014) 46-61.

[5] A. Faramarzi, M. Heidarinejad, B. Stephens, S. Mirjalili, Equilibrium optimizer: A novel optimization algorithm, Knowledge-Based Systems 191 (2020) 105190.

[6] S. Shadravan, H.R. Naji, V.K. Bardsiri, The Sailfish Optimizer: A novel nature-inspired metaheuristic algorithm for solving constrained engineering optimization problems, Engineering Applications of Artificial Intelligence 80 (2019) 20-34.

[7] G. Dhiman, V. Kumar, Spotted hyena optimizer: a novel bio-inspired based metaheuristic technique for engineering applications, Advances in Engineering Software 114 (2017) 48-70.

[8] F.A. Hashim, E.H. Houssein, M.S. Mabrouk, W. Al-Atabany, S. Mirjalili, Henry gas solubility optimization: A novel physics-based algorithm, Future Generation Computer Systems 101 (2019) 646-667.

[9] H. Yapici, N. Cetinkaya, A new meta-heuristic optimizer: Pathfinder algorithm, Applied Soft Computing 78 (2019) 545-568.

[10] S. Mirjalili, A.H. Gandomi, S.Z. Mirjalili, S. Saremi, H.Faris, S.M. Mirjalili, Salp Swarm Algorithm: A bio-inspired optimizer for engineering design problems. Advances in Engineering Software 114 (2017) 163-191 
[11] X.S. Yang, S. Deb, Engineering optimization by cuckoo search, International Journal of Mathematical Modelling and Numerical Optimisation, 1 (2010) 330-343.

[12] V.K. Patel, V.J. Savsani, Heat Transfer Search (HTS): a novel optimization algorithm, Information Sciences 324 (2015) 217-246

[13] Y.J. Zheng, Water wave optimization: a new nature-inspired metaheuristic, Computers \& Operations Research 55 (2015) 1-11

[14] V. Punnathanam, P. Kotecha, Yin-yang-pair optimization: A novel lightweight optimization algorithm, Engineering Applications of Artificial Intelligence 54 (2016) 62-79 [15] S. Mirjalili, The ant lion optimizer, Advances in Engineering Software 83 (2015) 80-98

[16] L. Cheng, X.H. Wu, Y. Wang, Artificial flora (af) optimization algorithm, Applied Sciences 8 (2018) 329

[17] S. Mirjalili, SCA: a sine cosine algorithm for solving optimization problems, Knowlegde-Based Systems 96 (2016) 120-133

[18] S. Mirjalili, Dragonfly algorithm: a new meta-heuristic optimization technique for solving single-objective, discrete, and multi-objective problems, Neural Computing \& Applications 27 (2016) 1053-1073

[19] A. Tabari, A. Ahmad, A new optimization method: Electro-Search algorithm, Computers \& Chemical Engineering 103 (2017) 1-11

[20] E. Jahani, M. Chizari, Tackling global optimization problems with a novel algorithmMouth Brooding Fish algorithm, Applied Soft Computing 62 (2018) 987-1002.

[21] https://www.ntu.edu.sg/home/epnsugan/index_files/cec-benchmarking.htm

[22] R. Storn, K. Price, Differential evolution - a simple and efficient heuristic for global optimization over continuous spaces, Journal of Global Optimization 11 (1997) 341-359.

[23] A.K. Qin, P.N. Suganthan, Self-adaptive differential evolution algorithm for numerical optimization, In IEEE congress on evolutionary computation, 2005, pp. 1785-1791. 
[24] R. Tanabe, A.S. Fukunaga, Improving the search performance of SHADE using linear population size reduction, In IEEE congress on evolutionary computation (CEC), 2014, pp. $1658-1665$.

[25] V.K. Patel, V.J. Savsani, M.A. Tawhid, Thermal System Optimization: A PopulationBased Metaheuristic Approach, Springer, Switzerland, 2019.

[26] K. Singh, R. Das, An experimental and multi-objective optimization study of a forced draft cooling tower with different fills, Energy Conversion and Management 111 (2016) 41730.

[27] K. Singh, R. Das, Multi Parameter Estimation in an Induced Draft Cooling Tower Using Genetic Algorithm, In Proceedings of the ASME 2016 International Mechanical Engineering Congress and Exposition, Phoenix, Arizona, USA, 2016, pp. 1-17.

[28] R.V. Rao, V.K. Patel, Optimization of mechanical draft counter flow wet-cooling tower using artificial bee colony algorithm, Energy Conversion and Management 52 (2011) 26112622.

[29] H. Ozcan, K. Ozdemir, H. Ciloglu, Optimum cost of an air cooling system by using differential evolution and particle swarm algorithms, Energy and buildings 65 (2013) 93-100. [30] M. Kintner-Meyer, A.F. Emery, Cost-optimal design for cooling towers. ASHRAE Journal 37 (1995) 46-55

[31] D. Joaquín, G. Salvador, M. Daniel, H. Francisco, A practical tutorial on the use of non parametric statistical tests as a methodology for comparing evolutionary and swarm intelligence algorithms, Swarm and evolutionary computation 1 (2011) 3-18

[32] R. Mallipeddi, P.N. Suganthan, Ensemble of Constraint Handling Techniques. IEEE Transactions on Evolutionary Computation, 14 (2010) 561-579. 
Table 1: Lower and upper limit of design variables and economic parameters

Geometric parameters

Water to air mass ratio, $\frac{m_{w}}{m_{a}}$

Mass velocity of water, $\frac{m_{w}}{A_{f i}}$

Mass velocity of air, $\frac{m_{a}}{A_{f i}}$

Capital cost factor, $\mathrm{a}_{\mathrm{cc}}$

Operating time, $Y_{h r}$

Electricity cost, $C_{e}$

Make-up water cost, $C_{w}$

Fixed cost, $C_{f}$

Incremental cost per unit mass flow, $C_{m a}$
Lower limit

1

Upper limit

10

10

1

$0.2983 /$ year

$8150 \mathrm{hr} /$ year

$0.085 \$ / \mathrm{kWh}$

$52.83 \times 10^{6} \$ / \mathrm{kg}$

$31185 \$$

$1097.5 \$ / \mathrm{kg} . \mathrm{s}^{-1}$ 
Table 2: Control parameters of the competitive algorithms

\section{SHO algorithm}

$\vec{h}=[5,0], \vec{M}=[0.5,1]$

MBF algorithm

$\mathrm{SP}=0.5, \mathrm{D}_{\text {is }}=1.5, \mathrm{P}_{\text {dis }}=0.5, \mathrm{SP}_{\text {damp }}=0.85$

SSA algorithm

$\mathrm{N}_{\mathrm{fs}}=4, \mathrm{G}_{\mathrm{c}}=1.9, \mathrm{P}_{\mathrm{dp}}=0.1$

SFO algorithm

$\mathrm{A}=4, \varepsilon=0.001$

PFA algorithm

$\alpha=1.2, \beta=1.4$

HHO algorithm,

$\mathrm{E}_{0}=[-1,1], \beta=1.5$

HGSO algorithm,

$\mathrm{M}_{1}=0.1, \mathrm{M}_{2}=0.2, \alpha=1, \beta=1, \mathrm{~K}=1$

MPA algorithm,

$\mathrm{P}=0.5, \mathrm{FADs}=0.2$

\section{EO algorithm}

$\mathrm{GP}=0.5, \lambda=[0,1], \mathrm{a}_{1}=2, \mathrm{a}_{2}=1$

DE algorithm

$\mathrm{CR}=0.5, \mathrm{~F}=0.5$

SaDE algorithm

CR \& F $=$ Self adapted

\section{L-SHADE algorithm}

$\mathrm{p}=0.11, \mathrm{H}=6, \mathrm{r}^{\mathrm{arc}}=2.6$ 
Table 3: Performance comparison of various algorithms

\begin{tabular}{llllll}
\hline Algorithms & Best value & Worst value & Mean value & Standard deviation & Success rate \\
\hline SHO & 61857.78 & 61926.22 & 61867.92 & $1.55 \mathrm{E}+01$ & 90 \\
MBF & 61863.15 & 61972.26 & 61876.6 & $2.67 \mathrm{E}+01$ & 81 \\
SSA & 61857.78 & 61926.22 & 61862.76 & $1.46 \mathrm{E}+01$ & 94 \\
SFO & 61858.04 & 61926.22 & 61869.69 & $1.93 \mathrm{E}+01$ & 89 \\
PFA & 61861.23 & 61941.56 & 61874.04 & $2.02 \mathrm{E}+01$ & 82 \\
HHO & 61857.78 & 61926.22 & 61866.71 & $1.56 \mathrm{E}+01$ & 91 \\
HGSO & 61863.9 & 61972.26 & 61878.37 & $2.90 \mathrm{E}+01$ & 79 \\
MPA & 61858.23 & 61926.22 & 61871.35 & $1.54 \mathrm{E}+01$ & 85 \\
EO & 61857.78 & 61926.22 & 61864.56 & $1.47 \mathrm{E}+01$ & 92 \\
DE & 61857.78 & 61864.2 & 61859.45 & $1.83 \mathrm{E}+00$ & 97 \\
SaDE & 61857.78 & 61864.2 & 61859.16 & $1.36 \mathrm{E}+00$ & 97 \\
L-SHADE & 61857.78 & 61864.2 & 61858.97 & $1.16 \mathrm{E}+00$ & 98 \\
\hline
\end{tabular}


Table 4: Statistical result of the comparative algorithms excluding DE and its variants

\begin{tabular}{llll} 
Algorithms & Freidman Value & Normalized Value & Rank of the algorithm \\
\hline SHO & 19 & 0.52632 & 4 \\
MBF & 40.5 & 0.24691 & 8 \\
SSA & 10 & 1 & 1 \\
SFO & 23 & 0.43478 & 6 \\
PFA & 35 & 0.28571 & 7 \\
HHO & 18 & 0.55556 & 3 \\
HGSO & 44.5 & 0.22472 & 9 \\
MPA & 22 & 0.45455 & 5 \\
EO & 13 & 0.76923 & 2 \\
\hline
\end{tabular}


Table 5: Statistical result of the comparative algorithms including DE, SaDE, and L-SHADE

\begin{tabular}{llll} 
Algorithms & Freidman Value & Normalized Value & Rank of the algorithm \\
\hline SHO & 31.5 & 0.66666 & 7 \\
MBF & 46.5 & 0.45161 & 11 \\
SSA & 28.5 & 0.73684 & 4 \\
SFO & 33.5 & 0.62686 & 9 \\
PFA & 43 & 0.48837 & 10 \\
HHO & 32.5 & 0.64615 & 8 \\
HGSO & 48.5 & 0.43299 & 12 \\
MPA & 30.5 & 0.68852 & 6 \\
EO & 29.5 & 0.71186 & 5 \\
DE & 23.5 & 0.89361 & 3 \\
SaDE & 21.5 & 0.97674 & 2 \\
L-SHADE & 21 & 1 & 1 \\
\hline
\end{tabular}


Table 6: Comparative results with various constraint handling methods

\begin{tabular}{|c|c|c|c|c|c|c|}
\hline Algorithms & & Static & Superiority of & Stochastic & $\varepsilon$-Constraint & Adaptive \\
\hline & Best & 61859.14 & 61858.83 & 61857.78 & 61858.83 & 61857.78 \\
\hline $\mathrm{SHO}$ & Mean & 61871.23 & 61869.63 & 61867.31 & 61868.73 & 61867.92 \\
\hline & SD & $1.81 \mathrm{E}+01$ & $1.71 \mathrm{E}+01$ & $1.54 \mathrm{E}+01$ & $1.61 \mathrm{E}+01$ & $1.55 \mathrm{E}+01$ \\
\hline & Best & 61864.22 & 61863.9 & 61863.36 & 61863.36 & 61863.15 \\
\hline MBF & Mean & 61879.83 & 61878.51 & 61877.32 & 61877.82 & 61876.6 \\
\hline & SD & $2.80 \mathrm{E}+01$ & $2.75 \mathrm{E}+01$ & $2.67 \mathrm{E}+01$ & $2.69 \mathrm{E}+01$ & $2.67 \mathrm{E}+01$ \\
\hline & Best & 61857.78 & 61857.78 & 61857.78 & 61857.78 & 61857.78 \\
\hline SSA & Mean & 61863.93 & 61863.27 & 61862.51 & 61863.13 & 61862.76 \\
\hline & SD & $1.52 \mathrm{E}+01$ & $1.48 \mathrm{E}+01$ & $1.39 \mathrm{E}+01$ & $1.32 \mathrm{E}+01$ & $1.46 \mathrm{E}+01$ \\
\hline & Best & 61859.14 & 61858.83 & 61858.34 & 61858.34 & 61858.04 \\
\hline SFO & Mean & 61874.72 & 61873.36 & 61870.85 & 61871.32 & 61869.69 \\
\hline & SD & $2.20 \mathrm{E}+01$ & $2.11 \mathrm{E}+01$ & $1.99 \mathrm{E}+01$ & $2.03 \mathrm{E}+01$ & $1.93 \mathrm{E}+01$ \\
\hline & Best & 61863.15 & 61862.44 & 61861.79 & 61861.79 & 61861.23 \\
\hline PFA & Mean & 61878.24 & 61876.66 & 61875.11 & 61875.82 & 61874.04 \\
\hline & SD & $2.33 \mathrm{E}+01$ & $2.23 \mathrm{E}+01$ & $2.08 \mathrm{E}+01$ & $2.13 \mathrm{E}+01$ & $2.02 \mathrm{E}+01$ \\
\hline & Best & 61858.23 & 61858.23 & 61857.78 & 61857.78 & 61857.78 \\
\hline $\mathrm{HHO}$ & Mean & 61868.75 & 61867.86 & 61866.93 & 61867.63 & 61866.71 \\
\hline & SD & $1.72 \mathrm{E}+01$ & $1.68 \mathrm{E}+01$ & $1.51 \mathrm{E}+01$ & $1.54 \mathrm{E}+01$ & $1.56 \mathrm{E}+01$ \\
\hline & Best & 61864.22 & 61864.22 & 61863.9 & 61863.9 & 61863.9 \\
\hline HGSO & Mean & 61882.04 & 61880.53 & 61879.21 & 61879.46 & 61878.37 \\
\hline & $\mathrm{SD}$ & $3.10 \mathrm{E}+01$ & $3.00 \mathrm{E}+01$ & $2.93 \mathrm{E}+01$ & $2.95 \mathrm{E}+01$ & $2.90 \mathrm{E}+01$ \\
\hline & Best & 61861.23 & 61860.72 & 61858.92 & 61858.92 & 61858.23 \\
\hline MPA & Mean & 61875.07 & 61873.12 & 61872.08 & 61872.77 & 61871.35 \\
\hline & SD & $1.91 \mathrm{E}+01$ & $1.79 \mathrm{E}+01$ & $1.60 \mathrm{E}+01$ & $1.61 \mathrm{E}+01$ & $1.54 \mathrm{E}+01$ \\
\hline
\end{tabular}




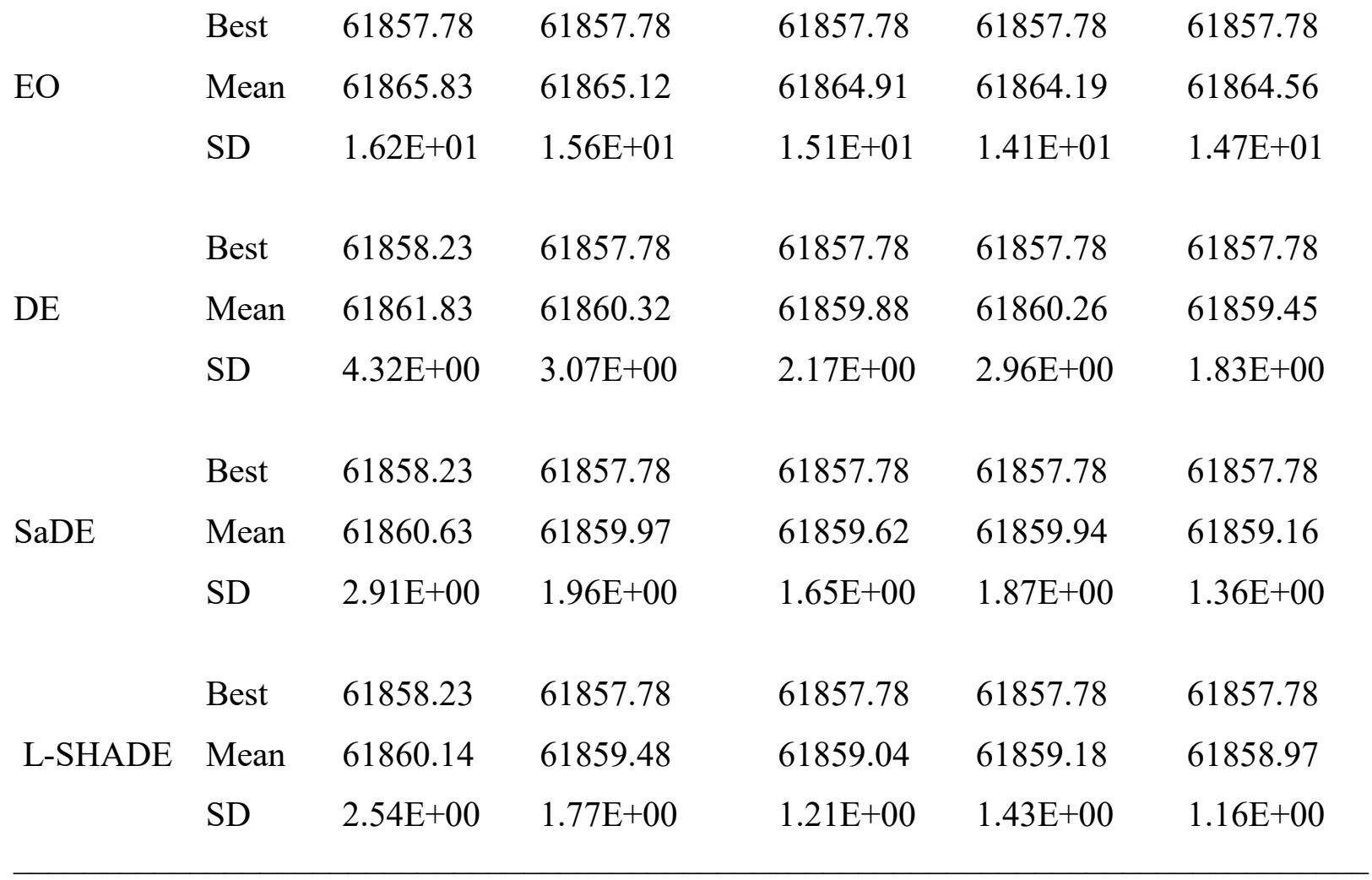


Table 7: Economically optimized geometry of cooling tower

Design Parameters Optimized value

Mass flow rate of water, $(\mathrm{kg} / \mathrm{s})$

32.49

Mass flow rate of air, $(\mathrm{kg} / \mathrm{s})$

17.45

Water-to-air mass ratio

1.861

Mass velocity of water, $\left(\mathrm{kg} / \mathrm{m}^{2} \mathrm{~s}\right)$

3.249

Mass velocity of air, $\left(\mathrm{kg} / \mathrm{m}^{2} \mathrm{~s}\right)$

1.746

Fill length of tower, (m)

3.93

Tower fill area, $\left(\mathrm{m}^{2}\right)$

10

Merkel number

5

Total Pressure drop, $(\mathrm{Pa})$

229.75

Total annual cost, (\$/year)

61857.78 
Table 8: Behaviour of competitive algorithms in respond to fill area constraints

\begin{tabular}{llllll}
\hline & $A_{f i}=9.6$ & $A_{f i}=9.8$ & $A_{f i}=10$ & $A_{f i}=10.2$ & $A_{f i}=10.4$ \\
\hline SHO & 61948.21 & 61903.54 & 61867.92 & 61847.17 & 61835.83 \\
MBF & 61952.43 & 61910.89 & 61876.6 & 61852.21 & 61840.74 \\
SSA & 61945.24 & 61896.68 & 61862.76 & 61842.59 & 61833.28 \\
SFO & 61947.12 & 61901.22 & 61869.69 & 61849.11 & 61837.79 \\
PFA & 61951.22 & 61910.47 & 61874.04 & 61851.89 & 61840.06 \\
HHO & 61946.76 & 61898.65 & 61866.71 & 61845.66 & 61834.93 \\
HGSO & 61955.88 & 61911.12 & 61878.37 & 61853.42 & 61842.41 \\
MPA & 61949.11 & 61906.59 & 61871.35 & 61850.44 & 61838.32 \\
EO & 61946.63 & 61897.68 & 61864.56 & 61843.45 & 61834.39 \\
DE & 61939.47 & 61892.12 & 61858.45 & 61838.92 & 61826.83 \\
SaDE & 61938.12 & 61891.88 & 61858.16 & 61837.19 & 61825.31 \\
L-SHADE & 61937.23 & 61891.18 & 61857.97 & 61836.43 & 61824.12 \\
\hline
\end{tabular}


Table 9: Behaviour of competitive algorithms in respond to Merkel number constraints

\begin{tabular}{llllll} 
Algorithms & \multicolumn{5}{c}{ Total annual cost, (\$) year) } \\
\hline & $M e=4.5$ & $M e=4.75$ & $M e=5$ & $M e=5.25$ & $M e=5.5$ \\
\hline SHO & 59190.22 & 60543.11 & 61867.92 & 63374.12 & 64839.92 \\
MBF & 59194.68 & 60547.79 & 61876.6 & 63381.34 & 64845.22 \\
SSA & 59188.93 & 60539.24 & 61862.76 & 63369.28 & 64835.37 \\
SFO & 59192.08 & 60544.53 & 61869.69 & 63377.48 & 64842.13 \\
PFA & 59193.36 & 60546.91 & 61874.04 & 63379.84 & 64844.77 \\
HHO & 59188.29 & 60541.07 & 61866.71 & 63372.88 & 64838.15 \\
HGSO & 59195.18 & 60549.85 & 61878.37 & 63382.09 & 64848.32 \\
MPA & 59192.87 & 60545.61 & 61871.35 & 63378.76 & 64843.91 \\
EO & 59187.79 & 60539.74 & 61864.56 & 63369.57 & 64836.14 \\
DE & 59183.84 & 60531.32 & 61858.45 & 63362.13 & 64827.96 \\
SaDE & 59181.95 & 60529.87 & 61858.16 & 63360.89 & 64826.34 \\
L-SHADE & 59180.63 & 60528.94 & 61857.97 & 63359.63 & 64824.27 \\
\hline
\end{tabular}




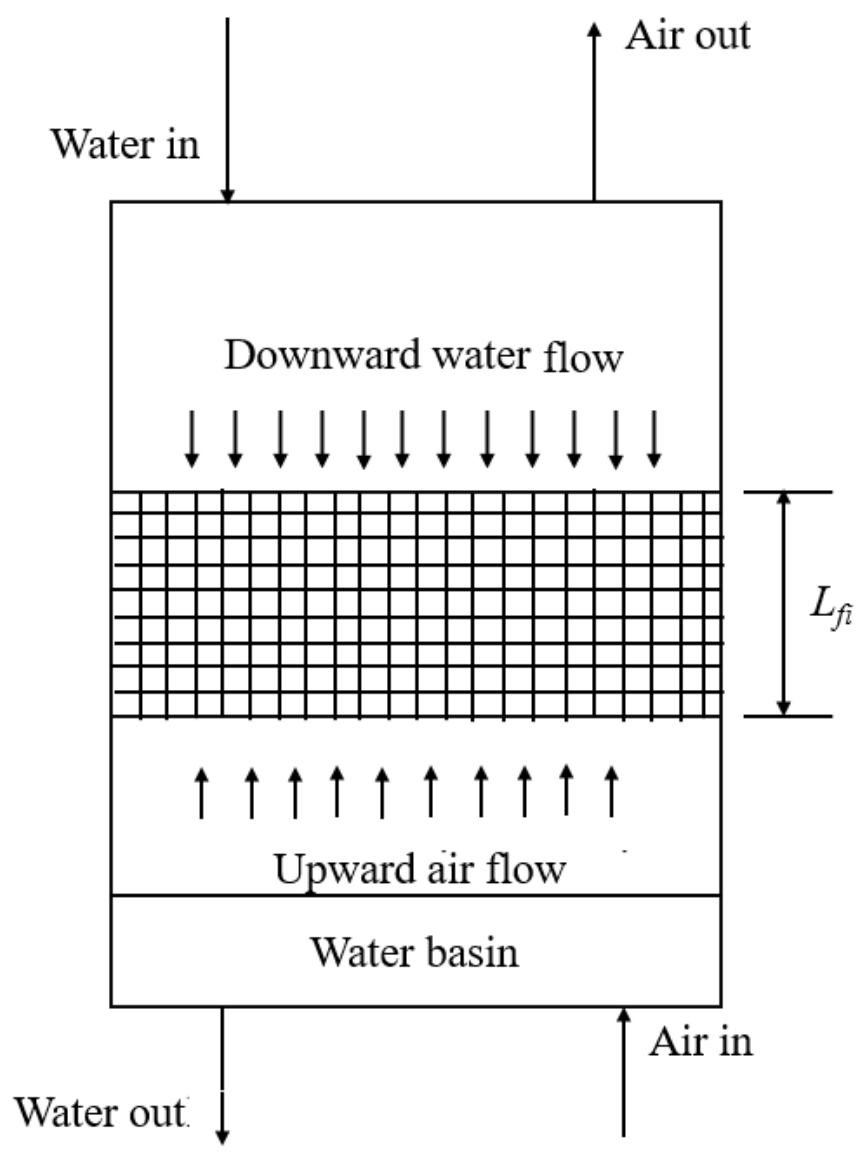

Fig 1: Geometry of mechanical draft counter flow cooling tower 


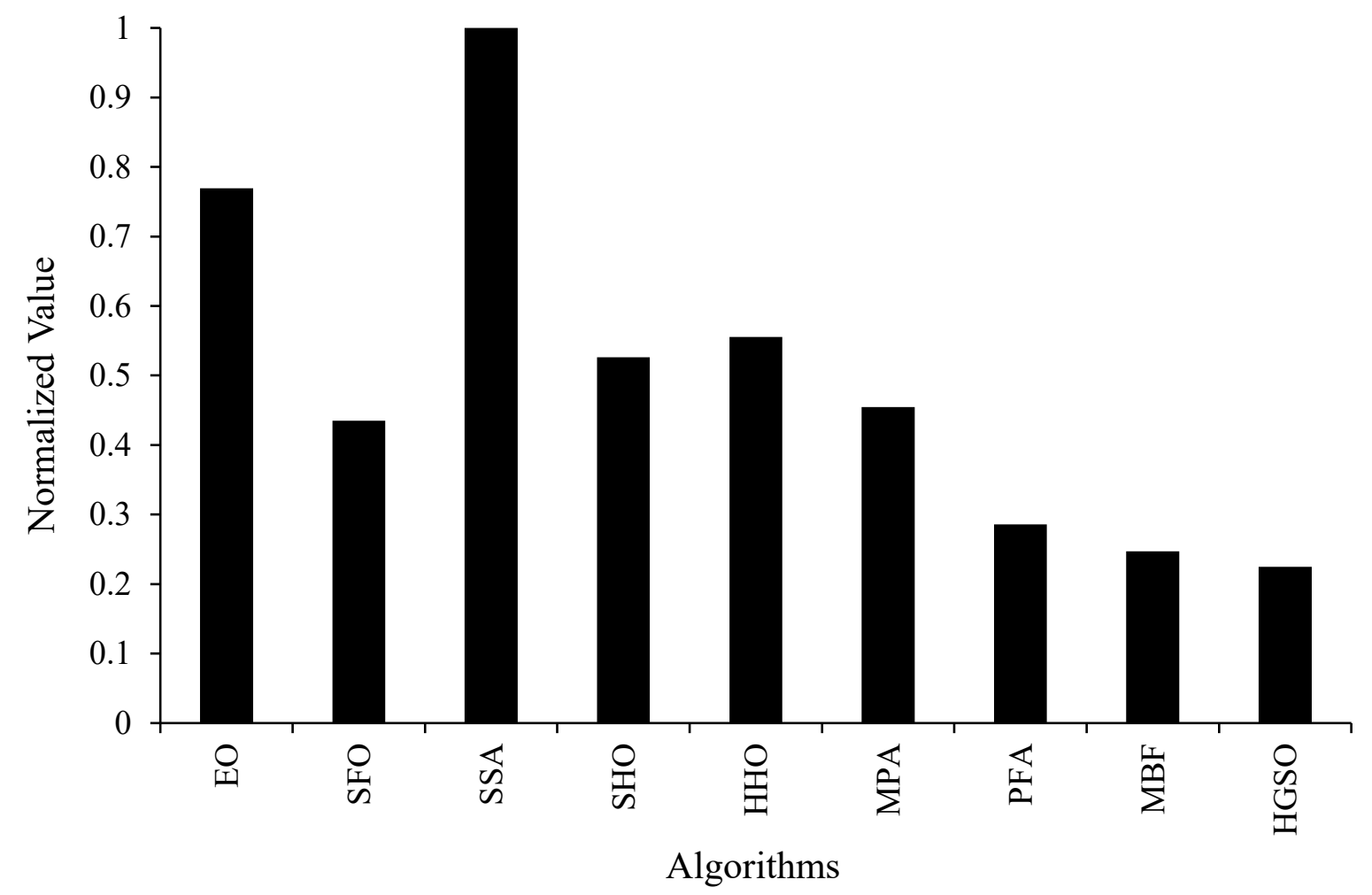

Fig. 2: Statistical result without considering DE, SaDE, and L-SHADE 


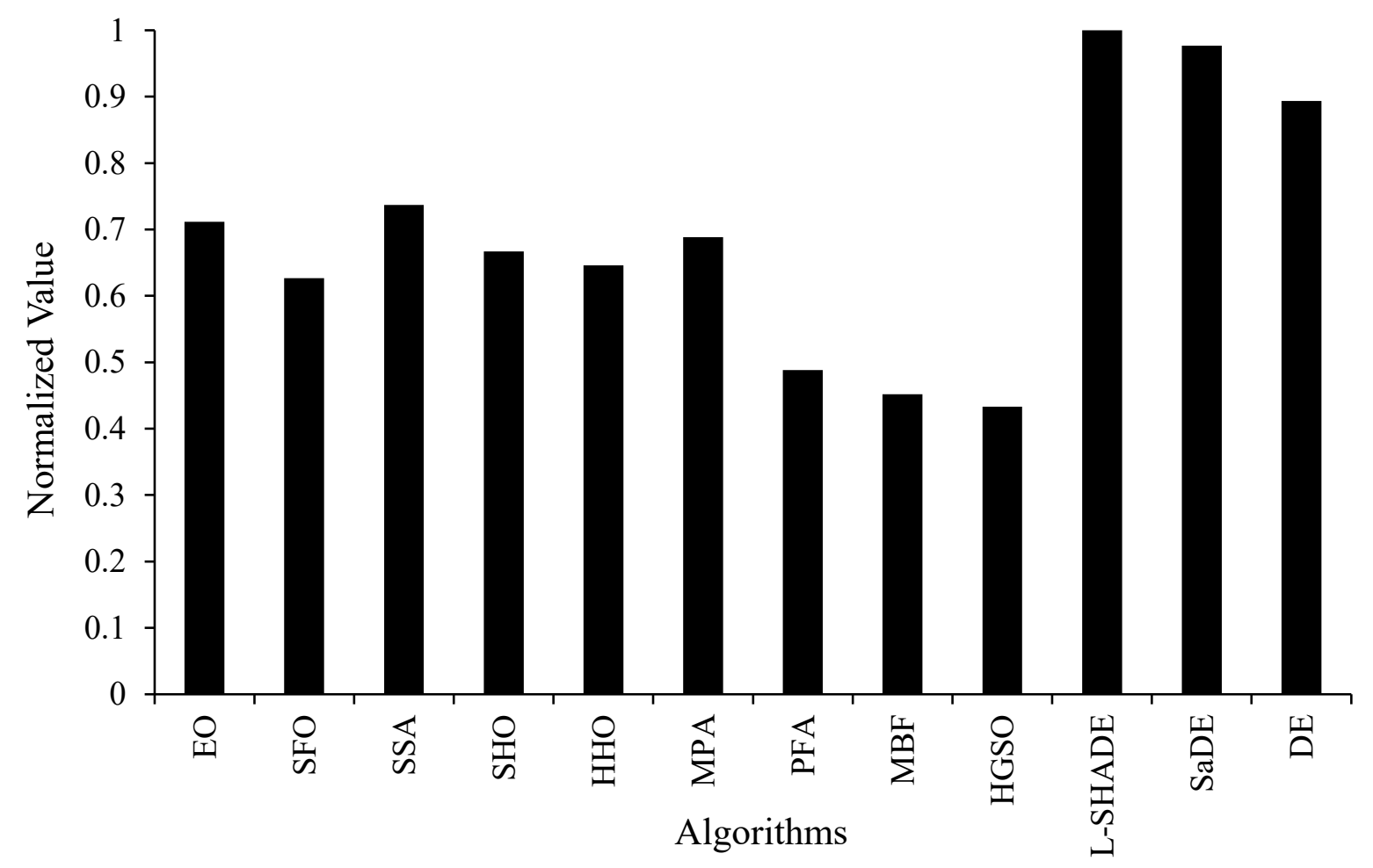

Fig. 3: Statistical result with DE, SaDE, and L-SHADE 


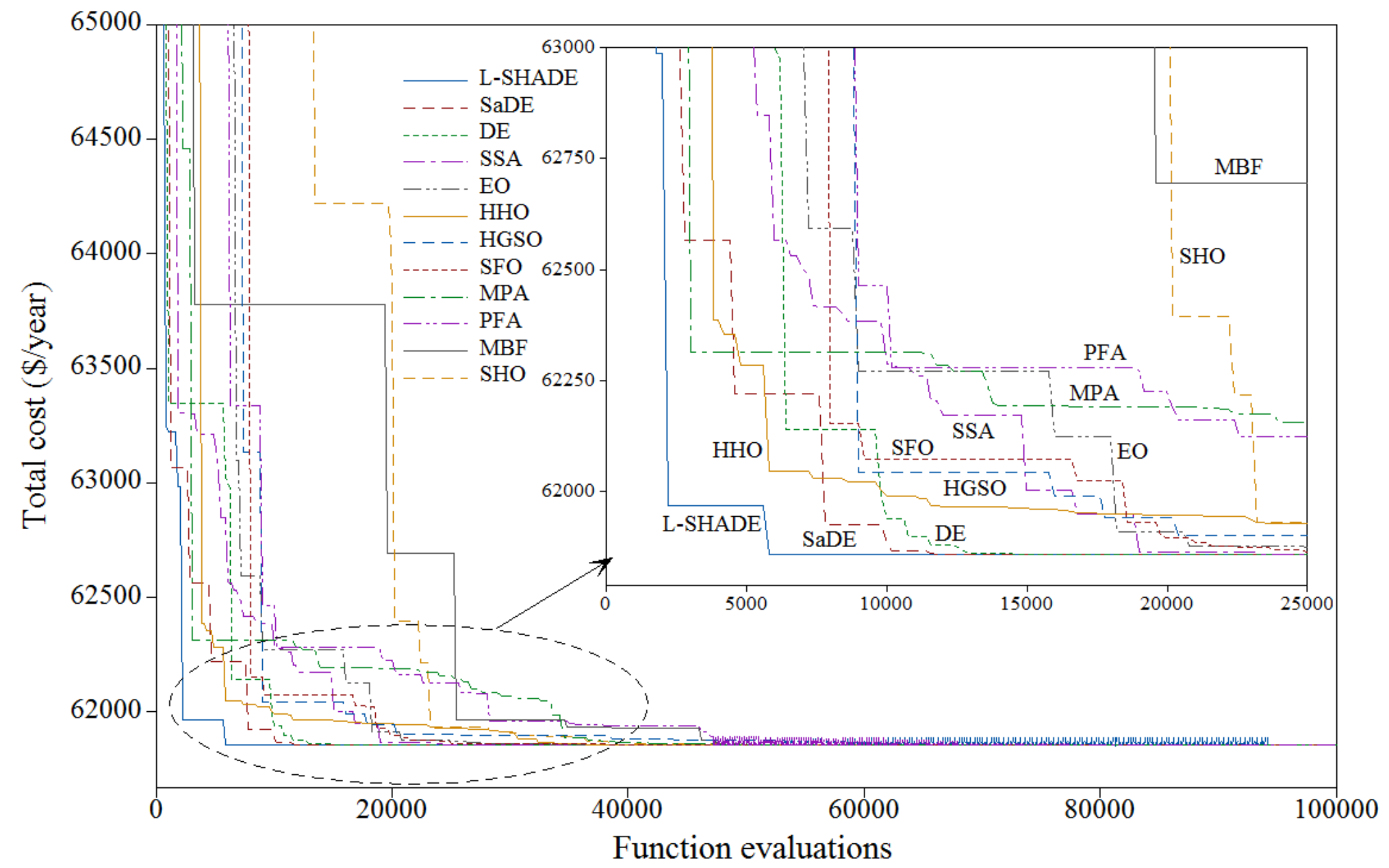

Fig. 4: Overall convergence comparison of comparative algorithms 

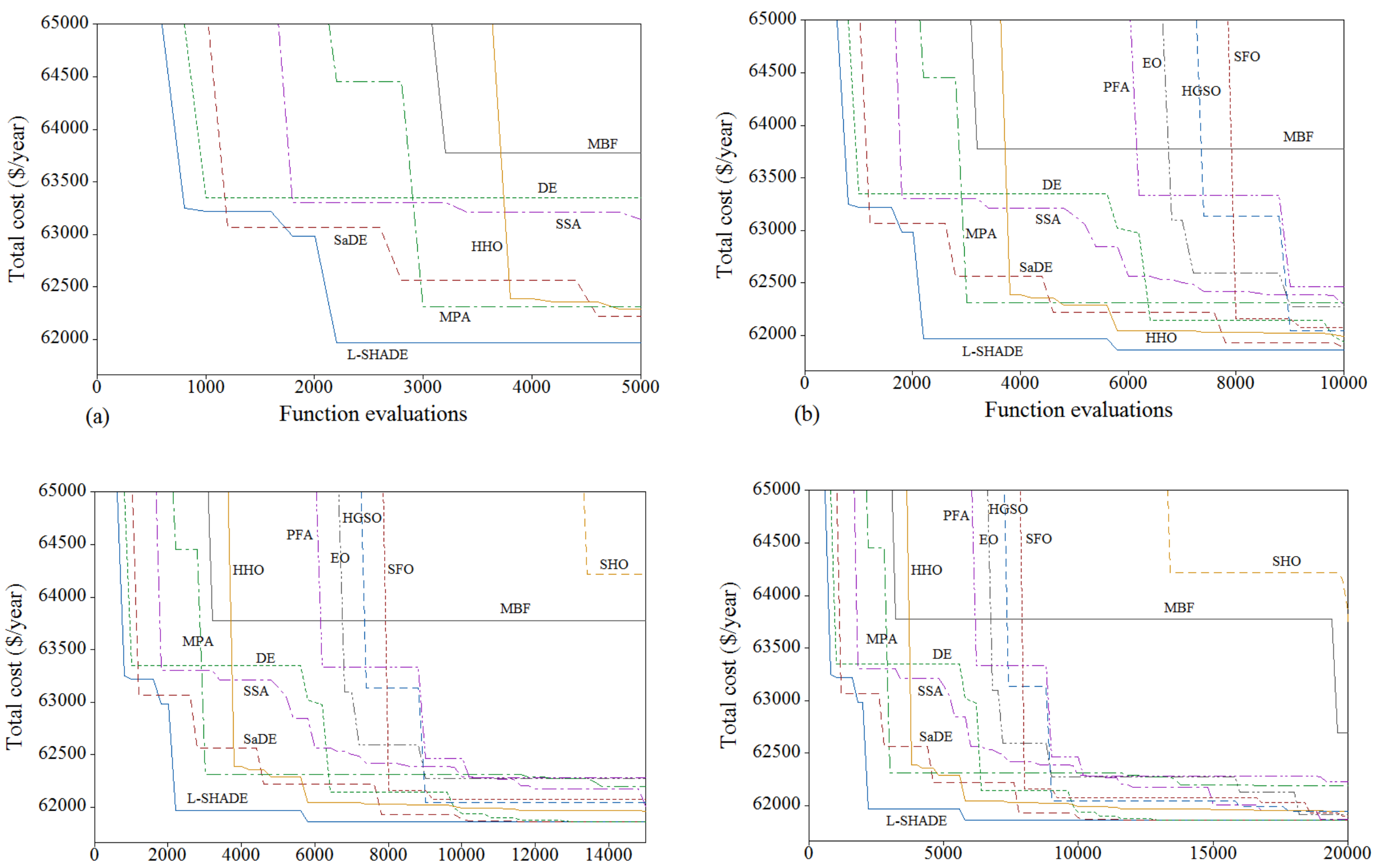

(c)

Function evaluations

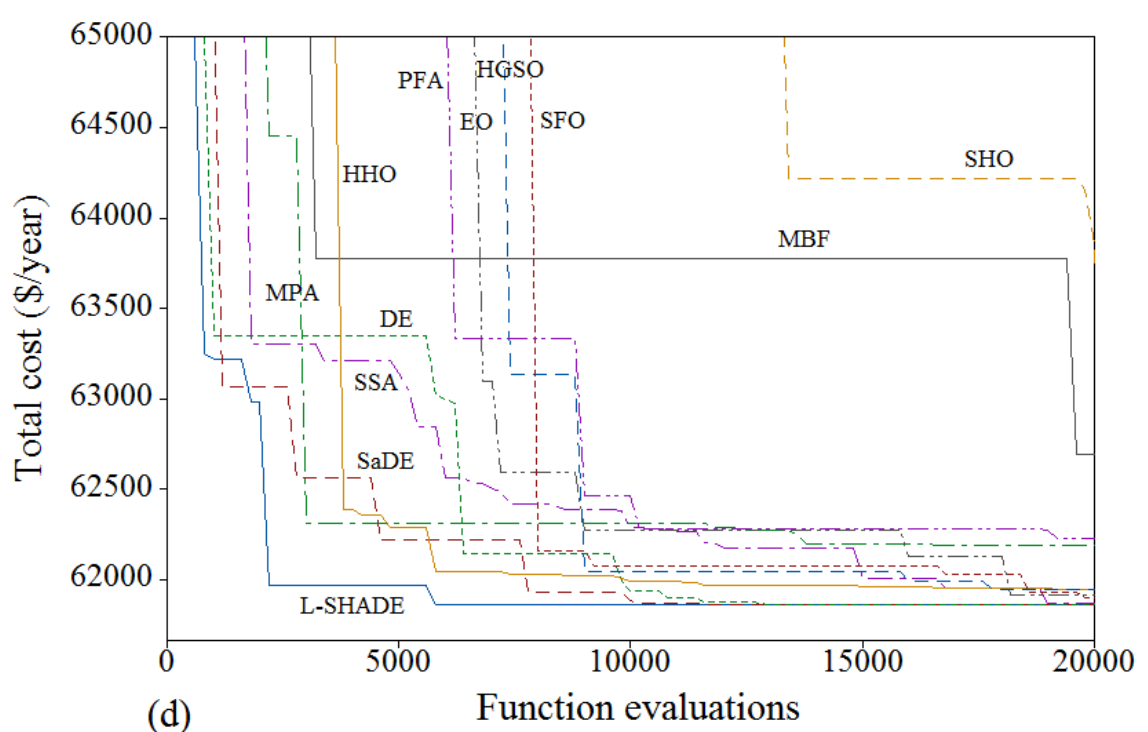

Fig. 5: Convergence behaviour of each competitive algorithm at the interval of (a) 5000 (b) 10000 (c) 15000 and (d) 20000 function evaluations 


\section{Appendix:}

Modelling equations for Mechanical draft cooling tower

Remarks

$Q=m_{w} c_{p w}\left(T_{\text {water,in }}-T_{\text {water }, \text { out }}\right)=m_{a}\left(h_{a, \text { out }}-h_{a, \text { in }}\right)$

$M e=\int_{T_{\text {water, out }}}^{T_{\text {wate }}} \frac{c_{p w} d T_{\text {water }}}{h_{s a}-h_{a}}=0.25 c_{p w}\left(T_{\text {water, in }}-T_{\text {water,out }}\right) \sum_{i-1}^{i-4} \frac{1}{\Delta h_{i}}$

$\Delta h_{i}=h_{s a, i}-h_{a, i}, \quad$ where $, i=1,2 \ldots .4$

$\Delta h_{a, i}=\Delta h_{a, i}+\frac{m_{w} c_{p w}}{m_{a}}\left(T_{\text {water }, \text { in }}-T_{\text {water }, \text { out }}\right), \quad i=1,2 \ldots .4$

$T_{\text {water }, i}=T_{\text {water,out }}+T C H_{i}\left(T_{\text {water, }, \text { in }}-T_{\text {water }, \text { out }}\right), \quad i=1,2 \ldots .4$

$h_{s a, i}=-6.388+0.865 T_{\text {water }, i}+15.715 \exp \left(0.05439 T_{\text {water }, i}\right), \quad i=1,2 \ldots .4$

$M e=\frac{h_{d} a_{f i} A_{f i} L_{f i}}{m_{w}}$

$\Delta P_{f i}=K_{f i} L_{f i} \frac{m a v_{m}^{2}}{2 \rho_{m} A_{f i}^{2}}$

$K_{f i}=\left[d_{1}\left(\frac{m_{w}}{A_{f i}}\right)^{d_{2}}\left(\frac{m_{a}}{A_{f i}}\right)^{d_{3}}+d_{4}\left(\frac{m_{w}}{A_{f i}}\right)^{d_{5}}\left(\frac{m_{a}}{A_{f i}}\right)^{d_{6}}\right] L_{f i}$

$m a v_{m}=\frac{m a v_{\text {in }}+m a v_{\text {out }}}{2}$
Heat transfer from water to air stream

Required Merkel number

Local enthalpy difference of air

Air enthalpy as per Chebyshev point

Water temperature as per Chebyshev point

Enthalpy of the saturated air

Available Merkel number

Fill matrix pressure drop

Loss coefficient correlations

Mean flow rate of air-vapor 
$\left\{m a v_{i n}=m_{a}+w_{i n} m_{a}\right.$

$\operatorname{mav}_{\text {out }}=\mathrm{m}_{\mathrm{a}}+\mathrm{w}_{\text {out }} \mathrm{m}_{\mathrm{a}}$

$\left\{\begin{array}{c}w_{\text {in }}=\left(\frac{2501.6-2.3263 W B T_{\text {air }, \text { in }}}{2506+1.8577 T_{\text {air }, \text { in }}-4.184 W B T_{\text {air }, \text { in }}}\right)\left(\frac{0.62509 P V_{w b t, i n}}{P_{\text {tot }}-1.005 P V_{w b t, i n}}\right) \\ -\left(\frac{1.00416\left(T_{\text {air }, \text { in }}-W B T_{\text {air }, \text { in }}\right)}{2506+1.8577 T_{\text {air }, \text { in }}-4.184 W B T_{\text {air }, \text { in }}}\right) \\ w_{\text {out }}=\frac{0.62509 P V_{\text {out }}}{P_{\text {tot }}-1.005 P V_{\text {out }}}\end{array}\right.$

$\Delta P_{m i s c}=K_{m c l} \frac{\rho_{m c l} v_{m c l}^{2}}{2}$

$P=\frac{\operatorname{mav}_{\text {in }} \Delta P_{t}}{\rho_{i n} \eta_{f}}$
Air-vapor flow rate at fill inlet \& outlet

Air humidity at inlet \& outlet

Miscellaneous pressure losses

Pumping power 


\section{Nomenclature used in Modelling}

$a_{f i} \quad$ area to volume ratio $\left(\mathrm{m}^{2} / \mathrm{m}^{3}\right)$

$A_{f i} \quad$ cooling tower fill area $\left(\mathrm{m}^{2}\right)$

$c_{p w} \quad$ specific heat of water $(\mathrm{J} / \mathrm{kg} \mathrm{K})$

$d_{1}-d_{6} \quad$ coefficients to estimation $K_{f i}$

$h_{a} \quad$ atmospheric air enthalpy $(\mathrm{J} / \mathrm{kg})$

$h_{s a} \quad$ saturated air enthalpy $(\mathrm{J} / \mathrm{kg})$

$h_{d} \quad$ coefficient of mass transfer $\left(\mathrm{kg} / \mathrm{m}^{2} \mathrm{~s}\right)$

$K_{f i} \quad$ loss coefficient

$L_{f i} \quad$ fill height (m)

$m_{a} \quad$ air mass flow $(\mathrm{kg} / \mathrm{s})$

$m_{w} \quad$ water mass flow rate $(\mathrm{kg} / \mathrm{s})$

mav air-vapor flow rate $(\mathrm{kg} / \mathrm{s})$

Me $\quad$ Merkel number

$P \quad$ pumping power required (W)

$P V \quad$ vapor pressure $(\mathrm{Pa})$

Q cooling duty (W)

$T \quad$ temperature $\left({ }^{\circ} \mathrm{C}\right)$

TCH Chebyshev constant

WBT atmospheric air wet-bulb temperature $\left({ }^{\circ} \mathrm{C}\right)$

$w \quad$ specific humidity $(\mathrm{kg} / \mathrm{kg}$ of air)

\section{Greek symbols}

$\Delta h \quad$ enthalpy difference $(\mathrm{J} / \mathrm{kg})$

$\Delta P_{t} \quad$ total pressure drop of the system $(\mathrm{Pa})$

$\eta_{f} \quad$ efficiency of fan 


$$
\rho \quad \text { density }\left(\mathrm{kg} / \mathrm{m}^{3}\right)
$$

\section{Subscripts}

$\begin{array}{ll}a & \text { air } \\ f i & \text { fill } \\ \text { in } & \text { inlet } \\ m & \text { mean } \\ m c l & \text { miscellaneous } \\ \text { out } & \text { outlet } \\ t & \text { total } \\ w & \text { water }\end{array}$

